\title{
Optimal Design of Remote Center Compliance Devices of Rotational Symmetry
}

\author{
Yang Liu and Michael Yu Wang* \\ Department of Mechanical and Automation Engineering, The Chinese University of Hong \\ Kong, Shatin, N.T., Hong Kong SAR, China \\ \{yliu2, yuwang\}@mae. cuhk. edu.hk
}

\begin{abstract}
Remote Center Compliance (RCC) devices are passive devices used in automated assembly. For round peg-in-hole insertions, initial misalignments of the peg can cause heavy radial loadings on the corresponding hole in arbitrary direction. RCC devices with rotational symmetry property are therefore desirable. This paper discusses how such devices may be designed and shows that circular periodic structures satisfy this property. The elastic part of the RCC device is formulated as a compliant mechanism and a systematic design methodology is proposed based on structural optimization. A smooth optimal design is achieved with distributed compliance and numerical simulation is conducted to illustrate the feasibility. The proposed method is expected to be used to design a novel device for assembling fragile plastic parts, which is a challenge to $3 \mathrm{C}$ industry.
\end{abstract}

Keywords: Remote Center Compliance (RCC), rotational symmetry, circular periodic structure, structural optimization.

\section{Introduction}

RCC devices were first developed at Draper Laboratory by Whitney and others [1-4]. Drake first pointed out that RCC devices could be created using rubber-metal sandwiches called elastomer shear pads (ESP) [3]. However, the analysis model for ESPs is inaccurate and it depends on the configuration parameters [5]. Havlik used three elastic rods to construct the device and proposed an algorithm to find rod parameters in [6]. Ciblak also investigated the possibility of using prismatic beams [7]. Drake and Hricko designed some devices conprising compliant linkages and elastic joints [2,8].

The elastic part of an RCC device can be modelled as a compliant mechanism. As lumped compliant mechanism will induce stress concentration, distributed compliant mechanism is desirable in actual applications. The kinetoelastic model which belongs to the continuum structural optimization method is an efficient tool of designing distributed compliance mechanisms and has been utilized here [9]. Rather than a fixed topology or shape configuration, structural optimization can significantly enlarge the design domain. Additionally, the optimization model could be applied to various

\footnotetext{
* Corresponding author. 
applications, such as assembly of small fragile parts in 3C industry, etc. It is therefore helpful to regard the design of an RCC device as question of structural optimization.

In the field of automated assembly, the task of fitting a round peg into a round hole is fundamental. As mentioned in the abstract, it is better to design a device with rotational symmetry property. The feasible structures that satisfy this property are discussed below. It has been proved that a circular periodic structure with at least 3 elements is workable and more elements are required if considering high-order geometric nonlinearities. The existing RCC device designs agree well with the above conclusions as most of them are circular symmetric structures with three or four elements.

The remainder of this paper is organized as follows: in Section 2, the compliant mechanism is analyzed and modeled using kinetoelastic model. The rotational symmetry property is introduced and feasible structures achieving this property are discussed. We show a schematic proof of the feasibility of the circular periodic structure. Section 3 presents formulation of the optimization model which includes the objective, constraints, design parameterization and solution scheme. The optimal design is presented and simulated in Section 4 to verify the proposed method. The conclusions and future works are discussed in Section 5.

\section{Design of RCC Devices Compliant Structure}

\subsection{Analysis of Compliant Mechanism}

Conventional and modified part mating events are shown in Fig. 1 and the shaded region is the design domain which can be modelled as a compliant mechanism. The second representation is adapted because it is easier to handle the structural optimization problem of compliant mechanism with a fixed upper boundary.
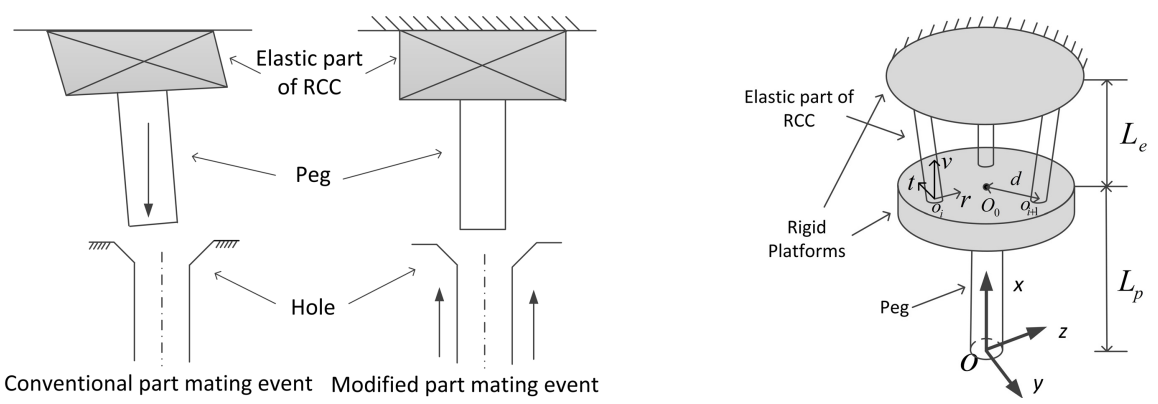

Fig. 1. Part mating events

Fig. 2. RCC Structure: placement and parameters

The compliant mechanism is formulated by the kinetoelastic model which concerns both kinematic motion and stiffness characteristics of compliant mechanism. According to Hooke's law, the force-displacement relationship at the rigid peg tip $O$ under global coordinate $O-X-Y-Z$ can be expressed as 


$$
K_{M} \cdot \Delta X=P
$$

where $K_{M}$ is the mechanism stiffness matrix, $P^{T}=\left[P_{x} P_{y} P_{z} M_{x} M_{y} M_{z}\right]$ and $\Delta X^{T}=\left[\Delta x \Delta y \Delta z \Delta \theta_{x} \Delta \theta_{y} \Delta \theta_{z}\right]$ are generalized force and motion at $O$ respectively. Notably, linear Hooke's law is satisfied due to the small clearance between peg and hole.

\subsection{Rotational Symmetry Design}

The rotational symmetry property is introduced in the abstract. Two kinds of feasible structures which satisfy this property are shown in Fig. 3. Note that revolutionary symmetric structure is occlusive. It costs much more in materials and its stiffness at radial direction would be exceptionally higher. Therefore, circular periodic structure is more reasonable.
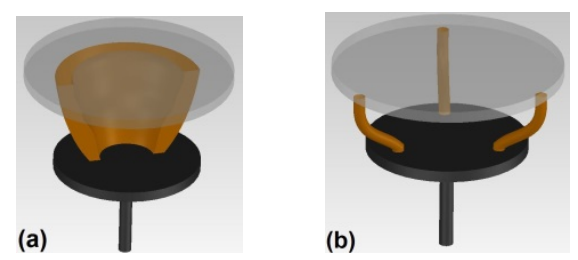

Fig. 3. Realization of rotational symmetry property: (a) revolutionary symmetric structure (only half is shown); (b) circular periodic structure

The schematic proof of circular periodic structure with $N$ elements can satisfy this property is described in the followings. Intermittently placed springs, shown in Fig. 4, can be utilized to represent the compliance elements. Each element comprises a radial spring (spring constant: $k$ ) and two tangential springs (spring constant: $k_{t}$ ). A small deformation $\delta$ in arbitrary direction $\theta$ is given at $O_{0}$.

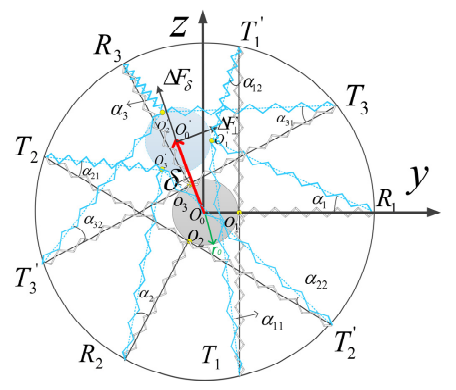

Fig. 4. Plan view of circular periodic structure (only three compliant elements are shown)

The resultant forces and directional stiffness in the direction of and perpendicular to $\delta$ together with the rotational moment and resultant stiffness can be obtained as

$$
\left\{\begin{array}{c}
\Delta F_{\delta}=\Delta F_{\delta r}+\Delta F_{\delta t 1}+\Delta F_{\delta t 2}=N\left(k+2 k_{t}\right) \delta / 2 \\
\Delta F_{\perp}=\Delta F_{\perp r}+\Delta F_{\perp t 1}+\Delta F_{\perp t 2}=0 \\
M_{r}=M_{t 1}=M_{t 2}=0
\end{array}\right.
$$




$$
\left\{\begin{array}{c}
K_{\delta}=\Delta F_{\delta} / \delta=N\left(k+2 k_{t}\right) / 2 \\
K_{\perp}=K_{\Delta \theta_{x}}=0
\end{array}\right.
$$

Combining the above results, the system stiffness $K$ is derived as

$$
K=N\left(k+2 k_{t}\right) / 2
$$

which is not related to the direction $\theta$. This completes the proof. Notably, the following trigonometric identities hold

$$
\left\{\begin{array}{c}
\sum_{i=1}^{N} \cos \left(\theta-\beta_{i}\right)=0 ; \sum_{i=1}^{N} \sin \left(\theta-\beta_{i}\right)=0 ;(N \geq 2) \\
\sum_{i=1}^{N} \cos \left[2\left(\theta-\beta_{i}\right)\right]=0 ; \sum_{i=1}^{N} \sin \left[2\left(\theta-\beta_{i}\right)\right]=0 ;(N \geq 3)
\end{array}\right.
$$

where $\beta_{i}=2 \pi(i-1) / N$. If second-order geometrical nonlinearity is considered, the following trigonometric identities should be utilized in the proof.

$$
\left\{\begin{array}{c}
\sum_{i=1}^{N}\left[\cos \left(\theta-\beta_{i}\right) \cdot \sin ^{2}\left(\theta-\beta_{i}\right)\right]=0 ;(N \geq 4) \\
\sum_{i=1}^{N} \sin ^{3}\left(\theta-\beta_{i}\right)=0 ;(N \geq 4)
\end{array}\right.
$$

Several interesting conclusions can be drawn about circular periodic structure and are summarized as: $(i)$ at least 3 discrete compliant elements are required; (ii)at least $N$ complaint elements are required if $(N-2)^{t h}(N \geq 4)$ order geometric nonlinearity is considered; (iii)when the number of complaint elements goes to infinity, the circular periodic structure becomes a revolutionary symmetric structure.

According to Hooke's law, the force-displacement relationship at beam tip $O_{i}$ under local coordinate $O_{i}-v-r-t$ is

$$
K_{E} \cdot \Delta X_{E}=P_{E}
$$

where $K_{E}$ is element stiffness matrix, $P_{E}$ and $\Delta X_{E}$ are generalized force and motion respectively. Notably, beam finite element is used to calculate $K_{E}$. With transformations of load and displacement, the mechanism stiffness matrix $K_{M}$ is achieved as

$$
K_{M}=\sum_{i=1}^{N} T_{i}^{O} \cdot K_{E} \cdot T_{i}^{O^{\prime}}
$$

where $T_{i}^{O}$ is the transformation matrix between local and global coordinates.

Suppose the distance between peg tip and compliance center is $a_{x}$. The mechanism stiffness matrix becomes diagonal at the compliance center, and $a_{x}$ can be acquired as

$$
a_{x}=-K_{M}(3,5) / K_{M}(3,3)=K_{M}(2,6) / K_{M}(2,2)
$$

Notably, the two ratios in Eq. 9 are equivalent according to Eq. 8. 


\section{Structural Optimization of RCC}

\subsection{Objective Function}

One practical optimization criterion is to maximize the output displacement $u_{o}$ which is the displacement of the contact point along the chamfer for unit normal contact force as shown in Fig. 5. According to geometric relationship equations, Eq. 1 and Eq. 8 , the objective function can be expressed as

$$
u_{o}=\Delta y / \cos \alpha=f_{1}(\mu)=A-\mu B
$$

where $\Delta y$ is the displacement of the peg tip, $\alpha$ is the angle of chamfer, $\mu$ is the coefficient of friction, $A$ and $B$ (positive) are constant coefficients. According to Eq. 10, $u_{o}$ is a decreasing function of $\mu$ which means that RCC device is more difficult to work under conditions of a higher coefficient of friction. Maximizing $u_{o}$ is equivalent to maintaining the same displacement at the output port under conditions of a higher coefficient of friction. Therefore, the objective function is equivalent to maximizing the range of friction coefficient that the RCC device can handle.

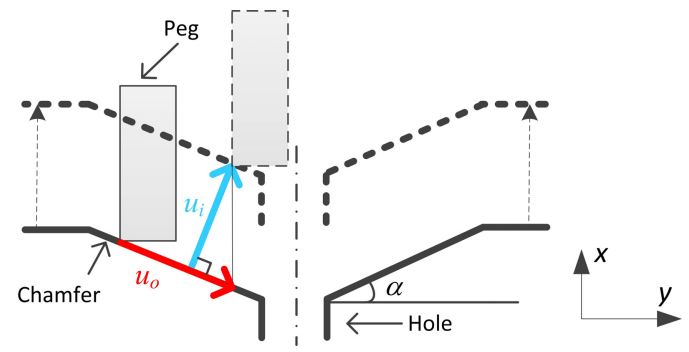

Fig. 5. Illustration of the chamfer crossing

\subsection{Design Constraints}

Successful Assembly Conditions. In order to cross the chamfer and avoiding wedging, the initial misalignments $\left(y_{h 0}, \theta_{h 0}\right)$ of the hole should satisfy the followings [4]:

$$
\left|y_{h 0}\right| \leq W ;\left|\theta_{h 0}\right| \leq\left(D_{h}-d_{p}\right) /\left(\mu d_{p}\right)
$$

where $W$ is width of chamfer, $D_{h}$ and $d_{p}$ are the diameters of hole and peg respectively. In order to avoid jamming, the applied forces must satisfy the followings [4]:

$$
\left\{\begin{array}{c}
-1 / \mu \leq P_{y} / P_{x} \leq 1 / \mu \\
-\lambda \leq M_{z} /\left(r_{p} P_{x}\right)+\mu(\lambda+1) P_{y} / P_{x} \leq \lambda
\end{array}\right.
$$

where $\lambda=l /\left(2 \mu r_{p}\right), r_{p}$ is the radius of peg and $l$ is the insertion depth. When $\lambda$ is small, jamming is most likely to happen and the slope of sides of the jamming diagram approaches $-\mu$. Thus, if the slope of applied force $-a_{x} / r_{p}$ is approximately equal to $-\mu$, then the applied forces and moments have the best chance of lying 
inside the jamming avoiding region [1]. Jamming constraint therefore can be converted into:

$$
0 \leq a_{x} \leq \mu r_{p}
$$

Constraint on Input Displacement. This is introduced to indirectly control the maximum stress levels in the compliant mechanism. For detailed explanations, please refer to reference [10]. The constraint of the input displacement $u_{i}$ is expressed as

$$
u_{i}=\Delta y \sin \alpha+\left(\Delta x-r_{p} \Delta \theta_{z}\right) \cos \alpha \leq\left[u_{i}\right]_{\max }
$$

where $u_{i}$ is the displacement of the contact point along chamfer's normal direction, which is shown in Fig. 5.

Constraint on Coupling Stiffness. Many coupling stiffness elements will vanish in $K_{M}$ due to periodic property. A large deformation would occur if the misalignment compensation capacity were increased. Then the elements in stiffness matrix will vary from each other and the coupling stiffness elements in $K_{M}$ will be non-zero which means the compliance center would drift away. It order to reduce its negative effect, the element of rotational stiffness about radial direction should be reduced to make the element like a spring with no coupling stiffness. From the perspective of engineering application, the ratio of two terms less than 0.1 can be regarded as small one and the numerator term can be ignored. We therefore introduce a constraint concerning the ratio of rotational stiffness about radial direction over tangential direction as

$$
S . R .=K_{E}(5,5) / K_{E}(6,6) \leq 0.1
$$

Constraint on Buckling and Stability. In order to maintain the stability of the compliant element, its critical buckling load should be greater than a given minimum value.

$$
P_{c r} \geq\left[P_{c r}\right]_{\min }
$$

\subsection{Model Formulation}

In conclusion, the optimization model can be summarized as follows.

$$
\left\{\begin{array}{c}
\text { Minimize: } J=-u_{o} \\
\left|y_{h 0}\right| \leq W \\
\text { Subject to: }\left\{\begin{array}{c}
\left|\theta_{h 0}\right| \leq\left(D_{h}-d_{p}\right) /\left(\mu d_{p}\right) \\
0 \leq a_{x} \leq \mu r_{p} \\
u_{i} \leq\left[u_{i}\right]_{\max } \\
S . R . \leq 0.1 \\
P_{c r}>\left[P_{c r}\right]_{\min } \\
K_{M} \cdot \Delta X=P
\end{array}\right.
\end{array}\right.
$$


The design variables are the distance $d=\overline{O_{0} O_{l}}$, shape $\Omega$ of compliant element cross section and neutral axis. The shape $\Omega$ at each vertical level is defined by a set of radial parameters which start from neutral axis to the surface. In this paper, two second order parabolic curves are employed to represent the neutral axis. The method of moving asymptotes (MMA) [11] is employed to solve the proposed optimization problem.

\section{$4 \quad$ RCC Device Design (Numerical Example)}

The design domain is defined as a cylinder with radius $R=100 \mathrm{~mm}$ and height $L_{e}=50$ $\mathrm{mm}$. The length of the peg and diameters of peg and hole are $100 \mathrm{~mm}, 12.000 \mathrm{~mm}$ and $12.033 \mathrm{~mm}$ respectively. The horizontal length and angle of chamfer are $3 \mathrm{~mm}$ and $\pi / 4$. The initial misalignments are given as $-3 \mathrm{~mm}$ and $0.0275 \mathrm{rad}$. The maximum input displacement is $0.2 \mathrm{~mm}$ and the minimum critical load is $1 \mathrm{KN}$. The Young's modulus, Poisson's ratio and coefficient of friction are $210 \mathrm{GPa}, 0.3$, and 0.1 respectively. In this paper, the RCC device with three compliant elements is designed to verify the proposed methodology. However, it is a straightforward matter to extrapolate to cover situations involving more than three elements. Note that the designed results are based on unit normal contact force.
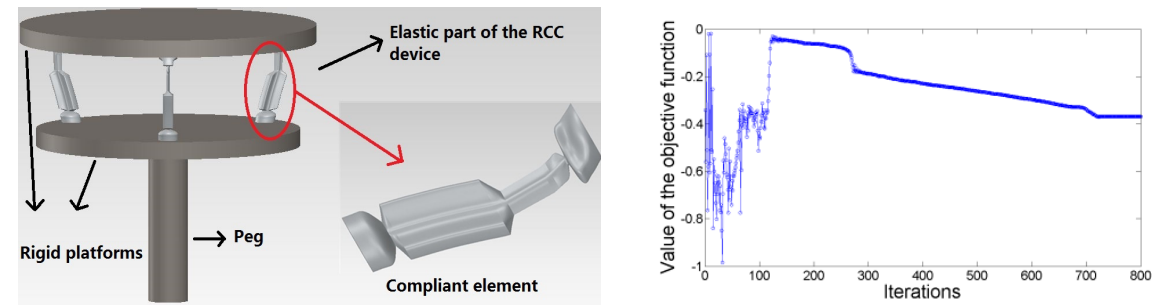

Fig. 6. Optimal design

Fig. 7. Iteration history of objective function

The optimal design is illustrated in Fig. 6. The optimal output and input displacements are $0.369 \mathrm{~mm}$ and $0.190 \mathrm{~mm}$ respectively. The position of the compliance center, the stiffness ratio and critical buckling load of optimal design are $0.138 \mathrm{~mm}$, 0.054 , and $1.682 \mathrm{KN}$ respectively. This clearly shows that all the constraints are satisfied. The optimal design is modelled and visualized with LOFT operation in SolidWorks by connecting all the optimal cross sections at different vertical levels. The optimal compliance element is quite smooth and flat mainly due to the constraint of coupling stiffness. Point flexures are avoided and distributed compliance is achieved by the proposed methodology. The convergence history of objective is illustrated in Fig. 7 which shows a good convergence. The present methodology generalizes some existing designs as the realization domain of compliant element is extended from round rod with elastic joints and round beam to beam with arbitrary cross sections.

The optimal design was analyzed and simulated by Autodesk Algor Simulation. The upper boundary of compliant mechanism is connected to a fixed platform and the 
lower boundary is joined to a very rigid platform. The property of remote compliance center is demonstrated in Fig. 8 where pure force results in a pure translation and pure moment causes a pure rotation. Numerical errors in translation and rotation are due to the small compliance in the platform and peg.
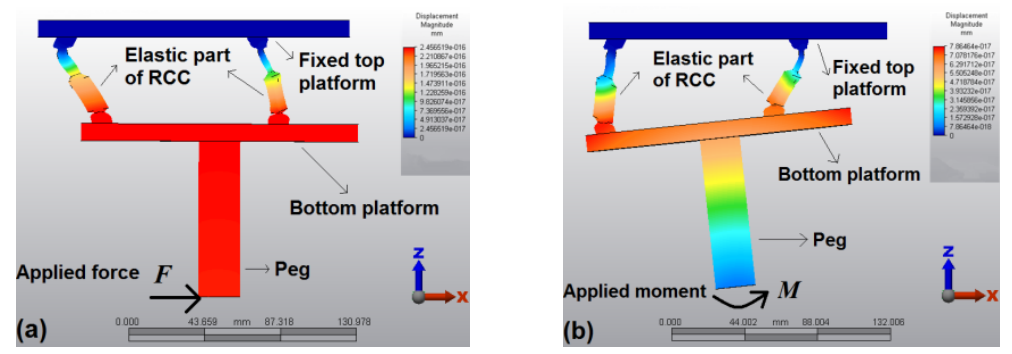

Fig. 8. Demonstration of compliance center: (a) pure translation, (b) pure rotation

\section{Conclusion}

We propose a novel design methodology by using structural optimization for design of an RCC device. The rotational symmetry property is discussed and it has been shown that a circular periodic structure satisfies this property. Comparing with revolutionary symmetric structure which is another feasible structure, circular periodic structure is adopted because it achieves quite low stiffness and saves on materials.

The elastic part of the RCC device was modelled as a compliant mechanism. To distribute the compliance, kinetoelastic model was utilized. The output displacement was selected as the objective and equivalent to the range of coefficient of friction that RCC device can handle. Beam finite element is utilized to analyze and simulate the complexity of design deformation. A smooth optimal design with distributed that is cost effective to manufacture is obtained in this paper. It satisfies all constraints and the properties of remote compliance center are demonstrated through the simulation.

The work reported here is far from complete. It is rewarding to integrate structural optimization with reliability constraint by considering the geometrical uncertainties of peg and hole, uncertainty of the coefficient of friction, etc. In addition, the discussed methodology can be applied to specified applications by introducing corresponding objective and constraints. For example, the applied force and vertical stiffness is critical in assembling fragile plastic parts which are easily damaged.

Acknowledgments. The authors would like to thank Prof. X. M. Wang, Prof. Y. J. Luo, Dr. L. Li and Dr. X. F. Tian for their informative discussions and comments, as well as Prof. K. Svanberg for providing his MMA codes. 


\section{References}

1. Whitney, D.: Mechanical Assemblies: Their Design, Manufacture, and Role in Product Development. Oxford University Press, New York (2004)

2. Drake, S.H.: Using Compliance in Lieu of Sensory Feedback for Automatic Assembly. Ph.D. Dissertation, MIT (1977)

3. Whitney, D., Rourke, J.M.: Mechanical Behavior and Equations for Elastomer Shear Pad Remote Center of Compliance. ASME J. Dyn. Syst. Meas. Control. 108, 223-232 (1986)

4. Whitney, D.: Quasi-static Assembly of Compliantly Supported Rigid Parts. ASME J. Dyn. Syst. Meas. Control. 104, 65-77 (1982)

5. Joo, S., Waki, H., Mayazaki, M.: On the Mechanics of Elastomer Shear Pads for Remote Center for Compliance (RCC). In: IEEE ICRA, pp. 291-298 (1996)

6. Havlik, S.: A New Elastic Structure for a Complaint Robot Wrist. Robotica. 1, 95-102 (1983)

7. Ciblak, N., Lipkin, H.: Design and Analysis of Remote Center of Compliant Structures. J. Robot. Syst. 20, 415-427 (2003)

8. Hricko, J., Havlík, S., Hart’anský, R.: Optimization in Designing Compliant Robotic Micro-Devices. In: Int. Workshop on Robotics in Alpe-Adria-Danube Region - RAAD, Hungary, pp. 397-402 (2010)

9. Wang, M.Y.: A Kinetoelastic Formulation of Compliant Mechanism Optimization. ASME J. Mech. Robot. 1, 021011.1-021011.10 (2009)

10. Sigmund, O.: On the Design of Compliant Mechanisms using Topology Optimization. Mech. Struct. Mach. 25, 493-524 (1997)

11. Svanberg, K.: The Method of Moving Asymptotes: a New Method for Structural Optimization. Int. J. Numer. Meth. Eng. 24, 359-373 (1987) 